\title{
On the Mathematical Relationship between Expected n-call@k and the Relevance vs. Diversity Trade-off
}

\author{
Kar Wai Lim \\ ANU \& NICTA \\ Canberra, Australia \\ karwai.lim@anu.edu.au
}

\author{
Scott Sanner \\ NICTA \& ANU \\ Canberra, Australia \\ ssanner@nicta.com.au
}

\author{
Shengbo Guo \\ Xerox Research Centre Europe \\ Grenoble, France \\ shengbo.guo@xrce.xerox.com
}

\begin{abstract}
It has been previously noted that optimization of the $n$ call@ $k$ relevance objective (i.e., a set-based objective that is 1 if at least $n$ documents in a set of $k$ are relevant, otherwise 0 ) encourages more result set diversification for smaller $n$, but this statement has never been formally quantified. In this work, we explicitly derive the mathematical relationship between expected $n$-call@k and the relevance vs. diversity trade-off — through fortuitous cancellations in the resulting combinatorial optimization, we show the trade-off is a simple and intuitive function of $n$ (notably independent of the result set size $k \geq n$ ), where diversification increases as $n \rightarrow 1$.
\end{abstract}

\section{Categories and Subject Descriptors}

H.3.3 [Information Search and Retrieval]: Retrieval Models

\section{Keywords}

diversity, set-based relevance, maximal marginal relevance

\section{RELEVANCE VS. DIVERSITY}

Subtopic retrieval — "the task of finding documents that cover as many different subtopics of a general topic as possible" [5] - is a motivating case for diverse retrieval. One of the most popular result set diversification methods is Maximal Marginal Relevance (MMR) 1]. Formally, given an item set $D$ (e.g., a set of documents) where retrieved items are denoted as $s_{i} \in D$, we aim to select an optimal subset of items $S_{k}^{*} \subset D$ (where $\left|S_{k}^{*}\right|=k$ and $k<|D|$ ) relevant to a given query q (e.g., query terms) with some level of diversity among the items in $S_{k}^{*}$. MMR builds $S_{k}^{*}$ in a greedy manner by choosing the next optimal selection $s_{k}^{*}$ given the set of $k-1$ optimal selections $S_{k-1}^{*}=\left\{s_{1}^{*}, \ldots, s_{k-1}^{*}\right\}$ (recursively defining $S_{k}^{*}=S_{k-1}^{*} \cup\left\{s_{k}^{*}\right\}$ with $\left.S_{0}^{*}=\emptyset\right)$ as follows:

$s_{k}^{*}=\underset{s_{k} \in D \backslash S_{k-1}^{*}}{\arg \max }\left[\lambda\left(\operatorname{Sim}_{1}\left(\mathbf{q}, s_{k}\right)\right)-(1-\lambda) \max _{s_{i} \in S_{k-1}^{*}} \operatorname{Sim}_{2}\left(s_{i}, s_{k}\right)\right]$.

Permission to make digital or hard copies of all or part of this work for personal or classroom use is granted without fee provided that copies are not made or distributed for profit or commercial advantage and that copies bear this notice and the full citation on the first page. To copy otherwise, to republish, to post on servers or to redistribute to lists, requires prior specific permission and/or a fee.

SIGIR'12, August 12-16, 2012, Portland, Oregon, USA.

Copyright 2012 ACM 978-1-4503-1472-5/12/08 ...\$15.00.

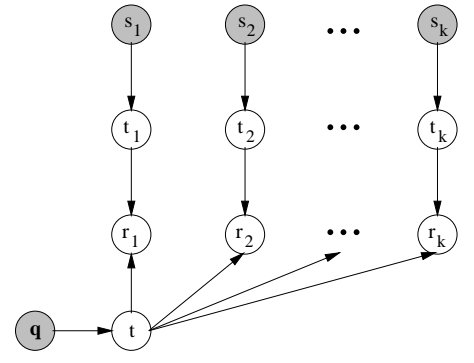

Figure 1: Latent subtopic binary relevance model.

Here, $\lambda \in[0,1]$, metric $\operatorname{Sim}_{1}$ measures query-item relevance, and metric $\mathrm{Sim}_{2}$ measures the similarity between two items.

Presently, little is formally known about how a particular selection of $\lambda$ relates to the overall set-based relevance objective being optimized. However, it has been previously noted that the $n$-call@ $k$ set-based relevance metric (which is 1 if at least $n$ documents in a set of $k$ are relevant, otherwise 0 ) encourages diversity as $n \rightarrow 1$ [2, 4. Indeed, Sanner et al. [3] have shown that optimizing expected $n$-call@ $k$ for $n=1$ corresponds to $\lambda=0.5$ - we extend this derivation to show that $\lambda=\frac{n}{n+1}$ for arbitrary $n \geq 1$ (independent of result set size $k \geq n)$. This result precisely formalizes a relationship between $n$-call@ $k$ and the relevance vs. diversity trade-off.

\section{RELEVANCE MODEL AND OBJECTIVE}

We review the probabilistic subtopic model of binary relevance from 3 shown as a directed graphical model in Figure1. Shaded nodes represent observed variables, unshaded nodes are latent. Observed variables are the query terms $\mathbf{q}$ and selected items $s_{i}$ (where for $1 \leq i \leq k, s_{i} \in D$ ). For the subtopic variables, let $T$ be a discrete subtopic set. Then $t_{i} \in T$ represent subtopics for respective $s_{i}$ and $t \in T$ represents a subtopic for query $\mathbf{q}$. The $r_{i}$ are $\{0,1\}$ variables that indicate if respective selected items $s_{i}$ are relevant $\left(r_{i}=1\right)$.

The conditional probability tables (CPTs) are as follows: $P\left(t_{i} \mid s_{i}\right)$ and $P(t \mid \mathbf{q})$ respectively represent the subtopic distribution for item $s_{i}$ and query q. For the $r_{i}$ CPTs, using $\mathbb{I}[\cdot]$ as a $\{0,1\}$ indicator function ( 1 if - is true), item $s_{i}$ is deemed relevant iff its subtopic $t_{i}$ matches query subtopic $t$ :

$$
P\left(r_{i}=1 \mid t, t_{i}\right)=\mathbb{I}\left[t_{i}=t\right]
$$

We next define $R_{k}=\sum_{i=1}^{k} r_{i}$, where $R_{k}$ is the number of relevant items from the first $k$ selections. Reading $R_{k} \geq n$ as $\mathbb{I}\left[R_{k} \geq n\right]$, we express the expected $n$-call@k objective as

$$
\operatorname{Exp}-n \text {-Call@ } k\left(S_{k}, \mathbf{q}\right)=\mathbb{E}\left[R_{k} \geq n \mid s_{1}, \ldots, s_{k}, \mathbf{q}\right] .
$$




\section{MAIN DERIVATION AND RESULT}

Taking MMR's greedy approach, we select $s_{k}$ given $S_{k-1}^{*} \mathbb{1}$

$$
\begin{aligned}
s_{k}^{*} & =\underset{s_{k}}{\arg \max } \mathbb{E}\left[R_{k} \geq n \mid S_{k-1}^{*}, s_{k}, \mathbf{q}\right] \\
& =\underset{s_{k}}{\arg \max } P\left(R_{k} \geq n \mid S_{k-1}^{*}, s_{k}, \mathbf{q}\right)
\end{aligned}
$$

This query can be evaluated w.r.t. our latent subtopic binary relevance model in Figure 1 as follows, where we marginalize out all non-query, non-evidence variables $T_{k}$ and define $T_{k}=$ $\left\{t, t_{1}, \ldots, t_{k}\right\}$ and $\sum_{T_{k}} \circ=\sum_{t} \sum_{t_{1}} \cdots \sum_{t_{k}} \circ$ :

$$
\begin{gathered}
=\underset{s_{k}}{\arg \max } \sum_{T_{k}}\left(P(t \mid \mathbf{q}) P\left(t_{k} \mid s_{k}\right) \prod_{i=1}^{k-1} P\left(t_{i} \mid s_{i}^{*}\right)\right. \\
\left.\cdot P\left(R_{k} \geq n \mid T_{k}, S_{k-1}^{*}, s_{k}, \mathbf{q}\right)\right)
\end{gathered}
$$

We split $R_{k} \geq n$ into two disjoint (additive) events $\left(r_{k} \geq\right.$ $\left.0, R_{k-1} \geq n\right),\left(r_{k}=1, R_{k-1}=n-1\right)$ where all $r_{i}$ are D-separated:

$$
\begin{aligned}
=\underset{s_{k}}{\arg \max } & \sum_{T_{k}} P(t \mid \mathbf{q}) P\left(t_{k} \mid s_{k}\right) \prod_{i=1}^{k-1} P\left(t_{i} \mid s_{i}^{*}\right) \\
& \cdot(\underbrace{P\left(r_{k} \geq 0 \mid R_{k-1} \geq n, t_{k}, t\right)}_{1} P\left(R_{k-1} \geq n \mid T_{k-1}\right) \\
& \left.+P\left(r_{k}=1 \mid R_{k-1}=n-1, t_{k}, t\right) P\left(R_{k-1}=n-1 \mid T_{k-1}\right)\right)
\end{aligned}
$$

We distribute initial terms over the summands noting that $\sum_{t_{k}} P\left(t_{k} \mid s_{k}\right) P\left(r_{k}=1 \mid t_{k}, t\right)=\sum_{t_{k}} P\left(t_{k} \mid s_{k}\right) \mathbb{I}\left[t_{k}=t\right]=P\left(t_{k}=t \mid s_{k}\right)$ :

$$
\begin{aligned}
& =\underset{s_{k}}{\arg \max }\left(\sum_{T_{k-1}}[\underbrace{\sum_{t_{k}} P\left(t_{k} \mid s_{k}\right)}] P\left(R_{k-1} \geq n \mid T_{k-1}\right) P(t \mid \mathbf{q}) \prod_{i=1}^{k-1} P\left(t_{i} \mid s_{i}^{*}\right)+\right. \\
& \left.\sum_{t} P(t \mid \mathbf{q}) P\left(t_{k}=t \mid s_{k}\right) \sum_{t_{1}, \ldots, t_{k-1}} P\left(R_{k-1}=n-1 \mid T_{k-1}\right) \prod_{i=1}^{k-1} P\left(t_{i} \mid s_{i}^{*}\right)\right)
\end{aligned}
$$

Next we proceed to drop the first summand since it is not a function of $s_{k}$ (i.e., it has no influence in determining $s_{k}^{*}$ ):

$$
=\underset{s_{k}}{\arg \max } \sum_{t} P(t \mid \mathbf{q}) P\left(t_{k}=t \mid s_{k}\right) P\left(R_{k-1}=n-1 \mid S_{k-1}^{*}\right)
$$

By similar reasoning, we can derive that the last probability needed in (2) is recursively defined as $P\left(R_{k}=n \mid S_{k}, t\right)=$

$$
\left\{\begin{aligned}
n \geq 1, k>1: & \left(1-P\left(t_{k}=t \mid s_{k}\right)\right) P\left(R_{k-1}=n \mid S_{k-1}, t\right) \\
& \quad+P\left(t_{k}=t \mid s_{k}\right) P\left(R_{k-1}=n-1 \mid S_{k-1}, t\right) \\
n=0, k>1: & \left(1-P\left(t_{k}=t \mid s_{k}\right)\right) P\left(R_{k-1}=0 \mid S_{k-1}, t\right) \\
n=1, k=1: & P\left(t_{1}=t \mid s_{1}\right) \\
n=0, k=1: & 1-P\left(t_{1}=t \mid s_{1}\right)
\end{aligned}\right.
$$

We can now rewrite (2) by unrolling its recursive definition. For expected $n$-call@ $k$ where $n \leq k / 2$ (a symmetrical result holds for $k / 2<n \leq k$ ), the explicit unrolled objective is

$$
\begin{aligned}
s_{k}^{*}=\underset{s_{k}}{\arg \max } \sum_{t}\left(P(t \mid \mathbf{q}) P\left(t_{k}=t \mid s_{k}\right) .\right. \\
\left.\sum_{j_{1}, \ldots, j_{n-1}} \prod_{l \in\left\{j_{1}, \ldots, j_{n-1}\right\}} P\left(t_{l}=t \mid s_{l}^{*}\right) \prod_{\substack{i=1 \\
i \notin\left\{j_{1}, \ldots, j_{n-1}\right\}}}^{k-1}\left(1-P\left(t_{i}=t \mid s_{i}^{*}\right)\right)\right)
\end{aligned}
$$

where $j_{1}, \ldots, j_{n-1} \in\{1, \ldots, k-1\}$ satisfy that $j_{i}<j_{i+1}$ (i.e., an ordered permutation of $n-1$ result set indices).

\footnotetext{
${ }^{1}$ We present a derivation summary; A full derivation may be found in an online appendix at the authors' web pages.
}

If we assume each document covers a single subtopic of the query (e.g., a subtopic represents an intent of an ambiguous query) then we can assume that $\forall i P\left(t_{i} \mid s_{i}\right) \in\{0,1\}$ and $P(t \mid \mathbf{q}) \in\{0,1\}$. This allows us to convert a $\prod$ to a $\max$

$$
\begin{aligned}
\prod_{\substack{i=1 \\
i \notin\left\{j_{1}, \ldots, j_{n-1}\right\}}}^{k-1}\left(1-P\left(t_{i}=t \mid s_{i}^{*}\right)\right) & =1-\left(1-\prod_{\substack{i=1 \\
i \notin\left\{j_{1}, \ldots, j_{n-1}\right\}}}^{k-1}\left(1-P\left(t_{i}=t \mid s_{i}^{*}\right)\right)\right) \\
& =1-\left(\max _{\substack{i \in[1, k-1] \\
i \notin\left\{j_{1}, \ldots, j_{n-1}\right\}}} P\left(t_{i}=t \mid s_{i}^{*}\right)\right)
\end{aligned}
$$

and by substituting this into (3) and distributing, we get

$$
\begin{gathered}
=\underset{s_{k}}{\arg \max } \sum_{t}\left(P(t \mid \mathbf{q}) P\left(t_{k}=t \mid s_{k}\right) \sum_{j_{1}, \ldots, j_{n-1}} \prod_{l \in\left\{j_{1}, \ldots, j_{n-1}\right\}} P\left(t_{l}=t \mid s_{l}^{*}\right)\right. \\
\left.-P(t \mid \mathbf{q}) P\left(t_{k}=t \mid s_{k}\right) \sum_{j_{1}, \ldots, j_{n-1}} \prod_{l \in\left\{j_{1}, \ldots, j_{n-1}\right\}} P\left(t_{l}=t \mid s_{l}^{*}\right) \max _{\substack{i \in[1, k-1] \\
i \notin\left\{j_{1}, \ldots, j_{n-1}\right\}}} P\left(t_{i}=t \mid s_{i}^{*}\right)\right) .
\end{gathered}
$$

Assuming $m$ selected documents $S_{k-1}^{*}$ are relevant then the top term (specifically $\left.\prod_{l}\right)$ is non-zero $\left(\begin{array}{c}m \\ n-1\end{array}\right)$ times. For the bottom term, it takes $n-1$ relevant $S_{k-1}^{*}$ to satisfy its $\prod_{l}$, and one additional relevant document to satisfy the $\max _{i}$ making it non-zero $\left(\begin{array}{c}m \\ n\end{array}\right)$ times. Factoring out the max element from the bottom and pushing the $\sum_{t}$ inwards (all legal due to the $\{0,1\}$ subtopic probability assumption) we get

$$
\begin{aligned}
\underset{s_{k}}{\arg \max } & \left(\begin{array}{c}
m \\
n-1
\end{array}\right) \underbrace{\sum_{t} P(t \mid \mathbf{q}) P\left(t_{k}=t \mid s_{k}\right)}_{\text {relevance: } \operatorname{Sim}_{1}\left(s_{k}, \mathbf{q}\right)} \\
& -\left(\begin{array}{c}
m \\
n
\end{array}\right) \operatorname{sax}_{s_{i} \in S_{k-1}^{*}} \underbrace{\sum_{t} P\left(t_{i}=t \mid s_{i}\right) P(t \mid \mathbf{q}) P\left(t_{k}=t \mid s_{k}\right)}_{\text {diversity: } \operatorname{Sim}_{2}\left(s_{k}, s_{i}, \mathbf{q}\right)} .
\end{aligned}
$$

From here we can normalize by $\left(\begin{array}{c}m \\ n-1\end{array}\right)+\left(\begin{array}{c}m \\ n\end{array}\right)=\left(\begin{array}{c}m+1 \\ n\end{array}\right)$ (Pascal's rule), leading to fortuitous cancellations and the result:

$=\underset{s_{k}}{\arg \max } \frac{n}{m+1} \operatorname{Sim}_{1}\left(s_{k}, \mathbf{q}\right)-\frac{m-n+1}{m+1} \max _{s_{i} \in S_{k-1}^{*}} \operatorname{Sim}_{2}\left(s_{k}, s_{i}, \mathbf{q}\right)$

Comparing to MMR in (1), we can clearly see that $\lambda=\frac{n}{m+1}$. Assuming $m \approx n$ since Exp-n-Call@k optimizes for the case where $n$ relevant documents are selected, then $\lambda=\frac{n}{n+1}$.

\section{Acknowledgements}

NICTA is funded by the Australian Government via the Dept. of Broadband, Comm. and the Digital Economy and the Australian Research Council through the ICT Centre of Excellence program.

\section{REFERENCES}

[1] J. Carbonell and J. Goldstein. The use of MMR, diversity-based reranking for reording documents and producing summaries. In SIGIR-98. ACM, 1998.

[2] H. Chen and D. R. Karger. Less is more: Probabilistic models for retrieving fewer relevant documents. In SIGIR-06. ACM, 2006.

[3] S. Sanner, S. Guo, T. Graepel, S. Kharazmi, and S. Karimi. Diverse retrieval via greedy optimization of expected 1-call@k in a latent subtopic relevance model. In CIKM-11. ACM, 2011.

[4] J. Wang and J. Zhu. Portfolio theory of information retrieval. In SIGIR-09. ACM, 2009.

[5] C. Zhai, W. W. Cohen, and J. Lafferty. Beyond independent relevance: Methods and evaluation metrics for subtopic retrieval. In SIGIR-03. ACM, 2003. 